\title{
Neurally adjusted ventilatory assist for infants in critical condition
}

\author{
"Given the experience from adult patients, and that leaks due to uncuffed \\ tubes are common in newborns, one could anticipate that patient-ventilator \\ asynchrony would have a high prevalence."
}

In the matter of 40 years, mechanical ventilation has developed from exclusively time-delivered controlled ventilation in rather heavily sedated (and often paralyzed) patients to neural control of mechanical ventilation in spontaneously breathing patients - that is, from 'being a slave of the ventilator to enslaving the ventilator.'

\section{Where do we come from?}

Although synchronized intermittent mandatory ventilation (SIMV) was released in the early 1980s, it was not before 2007 that the first peer-reviewed publication on SIMV and patient-ventilator asynchrony in infants was published [1]. The publication demonstrated that SIMV is a mode of ventilation that is not necessarily synchronized to the infant's breathing efforts and that mandatory breaths interfere with the breathing pattern. Despite the lack of evidence that the mode actually delivers what it promises, SIMV is frequently used and well accepted as one of the 'standard-of-care' modes of 'synchronized' mechanical ventilation to assist spontaneous breathing and to wean from mechanical ventilation.

"SIMV is a mode of ventilation that is not necessarily synchronized to the infant's breathing efforts and that mandatory breaths interfere with the breathing pattern."

Pressure support ventilation is another mode of mechanical ventilation that was developed in the early 1980s, and is also considered as a standard of care for spontaneous breathing, despite its limitations in terms of adequately synchronizing the ventilator's assist to breathing efforts [2].

In fact, recent literature demonstrate that more than a quarter of adult patients on pneumatically triggered standard-of-care modes of ventilation have severe asynchrony [3-6]. The asynchrony is more severe when assist levels are high [2], when sedation levels are high [6] and when leaks are present in the respiratory circuit, as with noninvasive ventilation [7]. Despite this growing evidence in adults, there are no studies evaluating the prevalence of patient-ventilator asynchrony in infants.

A good example of the lack of knowledge regarding the performance of a ventilator mode is the conclusion of the Cochrane database of systematic reviews for: synchronized mechanical ventilation for respiratory support in newborn infants [8]. "Compared with conventional ventilation, benefit is demonstrated for both highfrequency positive-pressure ventilation and triggered ventilation with regard to a reduction in air leak and a shorter duration of ventilation, respectively." Complex respiratory monitoring was not undertaken in any of the trials, thus it is not possible to conclude that the mechanism of producing those benefits is by provocation of synchronized ventilation.

Therefore, given the high prevalence of patient-ventilator asynchrony in adults, one can only anticipate the same - if not worse outcomes in infants who have higher respiratory rates, smaller tidal volumes, short time constants of the respiratory system, limited muscle strength and leaks due to the use of uncuffed endotracheal tubes.

\section{Does patient-ventilator asynchrony matter?}

A ventilator is a device that delivers respiratory support to a patient, and during so-called partial ventilatory assist it could be anticipated that the assist should be delivered when the patient is making their effort. If the patient's effort is rewarded by synchronized assist, the patient's effort and ventilator's assist occurring in tandem will allow for lower assist levels. During asynchrony, the portion of the patient's effort that is not effectively generating flow must be compensated by increasing the ventilator's assist level.
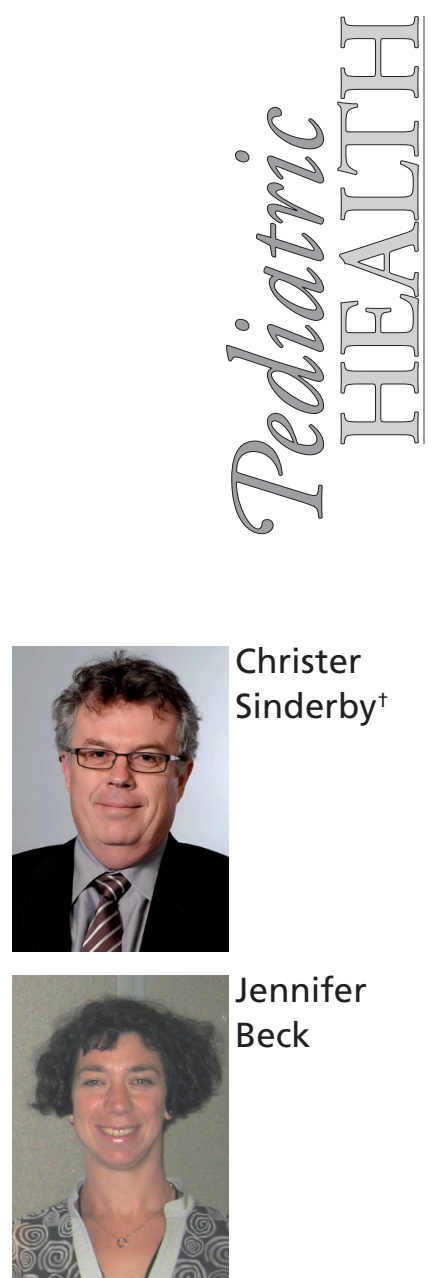

${ }^{+}$Author for correspondence: Keenan Research Centre in the Li Ka Shing Knowledge Institute of St Michael's Hospital, Toronto Department of Medicine, University of Toronto, 30 Bond Street, Queen Wing 4-072, Toronto, Ontario, Canada, M5B 1 W8

Tel.: +1 4168646060 ext. 2092

$+14168807507$

Fax: +1 4168645117

sinderbyc@smh.toronto.on.ca sinderby@rogers.com 
The most commonly reported form of patient ventilator asynchrony is when the patient's 'neural' respiratory rate is severely exceeding that registered and displayed by the ventilator, in other words, so-called wasted inspiratory efforts. Leung [2] and many others demonstrated that increasing assist levels with pneumatically triggered modes of mechanical ventilation increased the number of wasted inspiratory efforts. In other words, the ventilator monitor in pneumatically triggered modes underestimates the respiratory rate at high assist levels.

The most severe form of asynchrony is probably the combination of oversensitive trigger settings, high sedation levels and/or high assist levels, which can produce a situation resulting in an acceptable tidal volume and ventilator rate - misleading the clinician to believe that the patient is breathing despite the fact that the patient is suffering from a suppressed respiratory drive [9]. Inactivity of the respiratory muscles in both infants and adults leads to rapid and severe atrophy $[10,11]$, which may explain the severe weakness observed in mechanically ventilated patients deemed to be ready to be weaned [12].

In terms of patient outcome, negative consequences of patient-ventilator asynchrony in infants include decreased oxygenation [13,14], increased use of sedation or muscle paralysis [13-15], increased peak transpulmonary pressures [16] - which may put the infant at a higher risk for chronic lung disease - pneumothorax [17] and cerebral blood flow fluctuations, which can be associated with increased risk of intraventricular hemorrhage $[18,19]$.

"If the patient's effort is rewarded

by synchronized assist, the patient's effort and ventilator's assist occurring in tandem will allow for lower assist levels."

Although no studies have been performed in infants, studies in adult critical care patients have shown severe patient-ventilator asynchrony to be associated with prolonged time on mechanical ventilation [3], which, by definition, increases the risk of ventilator-associated pneumonia [20]. Patient-ventilator asynchrony worsens sleep quality in mechanically ventilated adult patients [21].

Literature in adult patients consistently shows that at least three factors have an impact on time of mechanical ventilation; this triad constitutes the necessity for allowing patients to breathe spontaneously [22]; the importance of limiting sedation [22]; and the prevention of patient-ventilator asynchrony [3]. Recently, the link between high levels of assist and time on ventilation was also confirmed in infants [23].

${ }^{6}$ NAVA introduced, for the first time, the ability of both monitoring the neural respiratory output to the diaphragm in relation to the assist delivery at the bedside, as well as delivering assist

in synchrony with the patient's neural inspiratory efforts."

This suggests that prolonged time on mechanical ventilation is due to a 'negative triad' of high sedation, high assist levels and severe asynchrony, where the use of sedation (to reduce agitation during, e.g., asynchrony) reduces respiratory drive and, thus, increases the need for assist, which in turn may aggravate the asynchrony and so on.

\section{The brain \& respiratory afferents control our breathing}

In 1966, Lourenco demonstrated that the diaphragm electromyogram (referred to as the diaphragm electrical activity [EAdi]) is proportional to phrenic nerve activity [24]. However, it was, in fact, not until 2000 that Sairam Parthasarathy and Martin Tobin's group at Loyola University of Chicago, IL, USA, evaluated the accuracy of flow, transdiaphragmatic pressure and diaphragm electromyography to predict synchrony between ventilator assist and a patient's neural effort [25]. It became clear that measures at the airway opening have severe limitations to determine patient-ventilator asynchrony. Our group was at the time involved in standardizing the signal processing of EAdi measured by catheters in the esophagus, resulting in a consensus for transesophageal EAdi measurements [26]. The new methodology for transesophageal EAdi measurements was adapted to be used for monitoring of respiratory drive [27] and for neural control of mechanical ventilation [28]. Finally, in 2007, neurally adjusted ventilatory assist (NAVA) was regulatory approved for all patient ages and released commercially. NAVA introduced, for the first time, the ability of both monitoring the neural respiratory output to the diaphragm in relation to the assist delivery at the bedside, as well as delivering assist in synchrony with the patient's neural inspiratory efforts. 


\section{Why use NAVA?}

To initiate this discussion, it should be emphasized that the sole purpose of our quest to develop neural monitoring and neural control of mechanical ventilation was to overcome the reported limitations of existing technologies. NAVA is a mode of ventilation that uses the EAdi to control the ventilator; however, monitoring the EAdi during conventional modes is also available.

\section{Monitoring}

The EAdi waveform - similar to the tidal volume, pressure or flow waveforms - can be monitored in real-time at the bedside during conventional modes of ventilation. This allows the clinician to question whether his/her patient is breathing at all times. The value of this pertains to the aforementioned studies highlighting the importance of spontaneous breathing in minimizing time on ventilation. By simply monitoring the presence of respiratory neural drive, it is possible to adjust sedation and/or levels of assist to ensure that inactivity is not forced upon the patient. The patient's respiratory response to different 'challenges' can be monitored to verify if appropriate respiratory reflexes are functioning. For example, reducing the pressure support level can be expected to cause an increase in the patients' respiratory drive [29].

\section{"By simply monitoring the presence of respiratory neural drive, it is possible to adjust sedation and/or levels of assist to ensure that inactivity is not forced upon the patient."}

By comparing the EAdi waveform with the airway pressure waveform (either by superimposing one signal on top of the other or by placement of time cursors), one now has the capability of monitoring patient-ventilator interaction and altering ventilator settings to optimize the synchrony (e.g., trigger or cycling-off criteria).

The EAdi waveform can be quantified in terms of its phasic (tidal) activity required to overcome respiratory loads, or the so-called 'tonic EAdi', which has recently been described in infants [30]. For example, breath-by-breath trending of the phasic EAdi over time might be an indication of the patient's ability to endure weaning. The tonic EAdi, or diaphragm activity present during exhalation, is a vagally mediated response responding to reductions in end-expiratory lung volume, by preventing derecruitment. By applying positive end-expiratory pressure, intubated infants respond by reducing the tonic EAdi, thereby allowing more 'rest' between phasic breaths.

The absence of the EAdi waveform is also informative and indicates central apnea. For apnea of premature, the EAdi has the potential to aid in therapeutic interventions aimed at reducing apnea (e.g., methylxanthine administration).

\section{NAVA}

Using the patient's own neural respiratory drive, NAVA controls the timing and assist of the ventilator [28]. Unlike conventional modes of ventilation that dictate the patients' respiratory drive, NAVA allows the patient's inherent respiratory feedback loops (via chemoreceptors, load receptors and vagal reflexes) to dictate the assist from the ventilator. This actually simplifies the implementation of mechanical ventilation since there is no need to guess which settings to use; the patient tailors the assist themselves. The cycling-off criteria are fixed at $70 \%$ of the peak of EAdi, however, are physiological since they are neurally based. Triggering during NAVA is based on a first-come, first-served principle (with pneumatic triggering) and runs with the basic settings. At a given NAVA level, the assist level adapts to the EAdi. Titration of the NAVA level has recently been evaluated, and it was demonstrated in critically ill patients that it is possible to determine when the respiratory demand is satisfied [31].

\section{Potential benefits of NAVA Improved synchrony}

Synchrony has two important components; first, the timing of assist delivery (start and stop of the breath), and second, the amount of assist provided in response to breath-by-breath changes in demand. Since the ventilator and the patient's diaphragm receive the same input from the brain (at the same time), NAVA is inherently synchronous to patient timing and effort. Colombo recently reported pressure support ventilation as being associated with severe asynchrony in 36\% of patients, whereas the prevalence with NAVA was $0 \%$ [5]. In premature infants, Beck et al. demonstrated that respiratory drive was suitable for controlling NAVA and was delivering assist in proportion to their efforts, while conventional ventilation provided fixed or unrelated changes in assist [32]. This allowance of spontaneous breathing patterns - and responding to them instantly - may have the potential benefit 
of allowing the infant to recruit their own lung (e.g., during sighs). It should be noted that, as in conventional ventilation, upper pressure limits can be set to prevent too high pressure delivery.

\section{Protection against overassist by downregulation of EAdi}

Inherent to NAVA is a neural respiratory afferent feedback. One very important reflex loop is the sensitivity of the lungs to stretch, mediated by the vagal nerves. The inflation-sensitive reflexes are very strong in infants, and will play an important role in delivery of assist with NAVA. In healthy adults, over distension of the lungs is limited during NAVA, even at extremely high NAVA levels [33]. In adult patients breathing on NAVA, it appears that tidal volumes are limited $[5,31]$ to values considered protective [34], regardless of the level of assist. Recent investigations in animals show that NAVA is at least as protective as current low-tidal-volume strategies [35]. In premature infants, the spontaneously chosen tidal volume is not in the injurious range [32].

\section{Synchrony is not affected by leaks}

Triggering and cycling-off of conventional noninvasive ventilation are often unreliable owing to leaks. The EAdi signal, being pneumatically independent, is not affected by leaks and can be used to control mechanical ventilation. In premature infants, synchrony (timing and proportionality) was not different during NAVA delivered invasively with an endotracheal tube or during NAVA delivered noninvasively with a single nasal prong [32].

\section{Conclusion}

The field of pediatric care in mechanical ventilation is clearly underdeveloped in that there is very little knowledge regarding the performance of mechanical ventilators in spontaneously breathing patients.

Given the experience from adult patients, and that leaks due to uncuffed tubes are common in newborns, one could anticipate that patientventilator asynchrony would have a high prevalence. To limit severe patient-ventilator asynchrony, future practice should resort towards a more careful selection of mode for ventilation, restriction of assist levels and adequate monitoring of patient-ventilator interaction. When applicable, sedatives should be used vigilantly.

\section{Financial \& competing interests disclosure}

Jennifer Beck and Christer Sinderby have made inventions related to neural control of mechanical ventilation that are patented. The license for these patents belongs to Maquet Critical Care. Future commercial uses of this technology may provide financial benefit to Jennifer Beck and Christer Sinderby through royalties. Jennifer Beck and Christer Sinderby each own 50\% of Neurovent Research Inc. (NVR). NVR is a research and development company that builds the equipment and catheters for research studies. NVR has a consulting agreement with Maquet Critical Care. The authors have no other relevant affiliations or financial involvement with any organization or entity with a financial interest in or financial conflict with the subject matter or materials discussed in the manuscript apart from those disclosed.

No writing assistance was utilized in the production of this manuscript.

7. Vignaux L, Vargas F, Roeseler J et al.: Patient-ventilator asynchrony during non-invasive ventilation for acute respiratory failure: a multicenter study. Intensive Care Med. 35(5), 840-846

(2009).

4. Thille AW, Cabello B, Galia F, Lyazidi A, Brochard L: Reduction of patient-ventilator asynchrony by reducing tidal volume during pressure-support ventilation. Intensive Care Med. 34(8), 1477-1486 (2008).

5. Colombo D, Cammarota G, Bergamaschi V et al.: Physiologic response to varying levels of pressure support and neurally adjusted ventilatory assist in patients with acute respiratory failure. Intensive Care Med. 34, 2010-2018 (2008).

6. de Wit M, Pedram S, Best AM, Epstein SK: Observational study of patient-ventilator asynchrony and relationship to sedation level. J. Crit. Care 24(1), 74-80 (2009).
8. Greenough A, Dimitriou G, Prendergast M, Milner AD: Synchronized mechanical ventilation for respiratory support in newborn infants. Cochrane Database Syst. Rev. 23(1), CD000456 (2008).

9. Sinderby C, Beck J: Proportional assist ventilation and neurally adjusted ventilatory assist - better approaches to patientventilator synchrony? Clin. Chest Med. 29(2), 329-342 (2008).

10. Levine $\mathrm{S}$, Nguyen $\mathrm{T}$, Taylor $\mathrm{N}$ et al.: Rapid disuse atrophy of diaphragm fibers in mechanically ventilated humans. N. Engl. J. Med. 358(13), 1327-1335 (2008). 
11. Knisely AS, Leal SM, Singer DB: Abnormalities of diaphragmatic muscle in neonates with ventilated lungs. J. Pediatr. 113(6), 1074-1077 (1988).

12. Laghi F, Cattapan SE, Jubran A et al:: Is weaning failure caused by low-frequency fatigue of the diaphragm? Am. J. Respir. Crit. Care Med. 167(2), 120-127 (2003).

13. Henry GW, Stevens CS, Schreiner RL, Grosfeld JL, Ballantine TVN: Respiratory paralysis to improve oxygenation and mortality in large newborn infants with respiratory distress. J. Pediatr. Surg. 14, 761-766 (1979).

14. Stark AR, Bascom R, Frantz ID: Muscle relaxation in mechanically ventilated infants. J. Pediatr. 94, 439-434 (1978).

15. Greenough A, Wood S, Morley CJ, Davis JA: Pancuronium prevents pneumothoraces in ventilated premature babies who actively expire against positive pressure ventilation. Lancet 1, 1-13 (1984).

16. Stark AR, Frantz ID: Prolonged expiratory duration with elevated lung volume in newborn infants. Pediatr. Res. 13, 261-264 (1979).

17. Greenough A, Morley CJ, Davis JA: Interaction of spontaneous breathing with artificial ventilation in pre-term babies. J. Pediatr. 103, 769-773 (1983).

18. Lipscomb AP, Reynolds EOR, Blackwell RJ et al: : Pneumothorax and cerebral haemorrhage in preterm infants. Lancet 414-416 (1981).

19. Perlman JM, Goodman S, Kreusser KL, Volpe JJ: Reduction in intraventricular hemorrhage by elimination of fluctuating cerebral blood-flow velocity in preterm infants with respiratory distress syndrome. N. Engl. J. Med. 312, 1353 -1357 (1985).

20. Srinivasan R, Asselin J, Gildengorin G, Wiener-Kronish J, Flori HR: A prospective study of ventilator-associated pneumonia in children. Pediatrics 123(4), 1108-1115 (2009).

21. Bosma K, Ferreyra G, Ambrogio C et al.: Patient-ventilator interaction and sleep in mechanically ventilated patients: pressure support versus proportional assist ventilation. Crit. Care Med. 35(4), 1048-1054 (2007).
22. Girard TD, Kress JP, Fuchs BD et al: Efficacy and safety of a paired sedation and ventilator weaning protocol for mechanically ventilated patients in intensive care (Awakening and Breathing Controlled trial): a randomised controlled trial. Lancet 371 , 126-134 (2008).

23. Traiber C, Piva JP, Fritsher CC et al.: Profile and consequences of children requiring prolonged mechanical ventilation in three Brazilian pediatric intensive care units. Pediatr. Crit. Care Med. 10(3), 375-380 (2009).

24. Lourenco RV, Cherniack NS, Malm JR, Fishman AP: Nervous output from the respiratory centers during obstructed breathing. J. Appl. Physiol. 21, 527-533 (1966).

25. Parthasarathy S, Jubran A, Tobin MJ: Assessment of neural inspiratory time in ventilator-supported patients. Am. J. Respir. Crit. Care Med. 162(2 Pt 1), 546-552 (2000).

26. Aldrich T, Sinderby C, McKenzie D, Estenne M, Gandevia S: Electrophysiologic techniques for the assessment of respiratory muscle function. ATS/ERS Statement on Respiratory Muscle Testing. Am. J. Respir. Crit. Care Med. 166, 518-624 (2002).

27. Beck J, Sinderby C, Lindström L, Grassino A: Effects of lung volume on diaphragm EMG signal strength during voluntary contractions. J. Appl. Physiol. 85, 1123-1134 (1998).

28. Sinderby C, Navalesi P, Beck J et al.: Neural control of mechanical ventilation in respiratory failure. Nat. Med. 5, 1433-1436 (1999).

-• First and original paper describing neurally controlled mechanical ventilation and neural monitoring.

29. Beck J, Gottfried SB, Navalesi P et al:: Electrical activity of the diaphragm during pressure support ventilation in acute respiratory failure. Am. J. Respir. Crit. Care Med. 164(3), 419-424 (2001).

30. Emeriaud G, Beck J, Tucci M, Lacroix J, Sinderby C: Diaphragm electrical activity during expiration in mechanically ventilated infants. Pediatr. Res. 59, 705-710 (2006).
31. Brander L, Leong-Poi H, Beck J et al.: Titration and implementation of neurally adjusted ventilatory assist in critically ill patients. Chest 135(3), 695-703 (2009).

32. Beck J, Reilly M, Grasselli G et al.: Patient-ventilator interaction during neurally adjusted ventilatory assist in low birth weight infants. Pediatr. Res. 65(6), 663-668 (2009).

- First paper to demonstrate that the neural respiratory drive in preterm infants is suitable to control mechanical ventilation.

33. Sinderby C, Beck J, Spahija J et al:: Inspiratory muscle unloading by neurally adjusted ventilatory assist during maximal inspiratory efforts in healthy subjects. Chest 131, 711-717 (2007).

34. ARDS Network: Ventilation with lower tidal volumes as compared with traditional tidal volumes for acute lung injury and the acute respiratory distress syndrome. $N$. Engl. J. Med. 18, 1301-1308 (2000).

35. Brander L, Sinderby C, Lecomte F et al.: Neurally adjusted ventilatory assist decreases ventilator inducedlung injury and nonpulmonary organ dysfunction in rabbits with acute lung injury. ICM (In Press) (2009).

\section{Affiliations}

- Christer Sinderby

Keenan Research Centre in the Li Ka Shing Knowledge Institute of St Michael's Hospital, Toronto Department of Medicine, University of Toronto, 30 Bond Street, Queen Wing 4-072, Toronto, Ontario, Canada, M5B 1W8 Tel.: +1 4168646060 ext. 2092

$$
+14168807507
$$

Fax: +1 4168645117

sinderbyc@smh.toronto.on.ca sinderby@rogers.com

- Jennifer Beck

Keenan Research Centre in the Li Ka Shing Knowledge Institute of St Michael's Hospital, Toronto Department of Medicine, University of Toronto, 30 Bond Street, Queen Wing 4-072, Toronto, Ontario, Canada, M5B 1W8 Tel.: +1 4168646060 ext. 2092

$+14168803664$

Fax: +1 4168645117

beckj@smh.toronto.on.ca jennifer.beck@rogers.com 